\title{
Impacto del turismo rural sobre el empleo en España: una
}

aproximación a escala provincial*

Cómo citar este artículo: Sánchez Sánchez, A., y Sánchez Sánchez, F. (2018). Impacto del turismo rural sobre el empleo en España: una aproximación a escala provincial. Cuadernos de Desarrollo Rural, I5(82), I-I9. https://www.doi.org/I0.III44/Javeriana.cdri5-82.itre

Ana María Sánchez Sánchez

Universidad Pablo de Olavide, España

amsansan@upo.es

ORCID: http://orcid.org/0000-0002-659I-954X

Francisca Jesús Sánchez

Universidad Pablo de Olavide, España

ORCID: http://orcid.org/0000-000I-5325-3667

DOI: https://doi.org/I0.III44/Javeriana.cdr15-82.itre

Redalyc: http://www.redalyc.org/articulo.oa?id=II757724002

Recibido: 20 Marzo 2018 I Publicación: I8 Diciembre 2018

\section{Resumen:}

El turismo rural es una actividad con una importancia creciente en el entorno rural, pues ayuda a complementar la renta agraria e incluso a mitigar la despoblación de estas zonas. El turismo rural debe ser entendido como una opción económica importante para las zonas rurales y como un eje estratégico para el desarrollo territorial que ayuda al desarrollo de áreas rurales que cuentan con una mala situación económica y social. Por ello, se estudia este turismo como alternativa económica de revitalización y desarrollo en las zonas rurales, por medio del análisis del impacto de esta actividad en el empleo del entorno. Se analizan variables que recogen información sobre tres bloques temáticos: economía, demografía y turismo rural. Para el análisis de datos, se aplican el análisis factorial, el análisis clúster y el análisis discriminante. Se extraen tres factores: dinamismo turístico-laboral, dinamismo turístico-rural y turismo de calidad versus provincias "deprimidas" laboralmente. De acuerdo con estos factores, se realiza una clasificación de las provincias españolas en cuanto al impacto del turismo rural en el empleo, en tres grupos, que demostró una influencia desigual dependiendo de la provincia. Asturias es la provincia española donde el turismo rural tiene un mayor impacto positivo sobre el empleo. En contraposición, en las provincias de Cádiz, Málaga, Las Palmas y Santa Cruz de Tenerife, a pesar de que tienen un turismo rural que busca la calidad medioambiental que ofrecen los espacios protegidos, este turismo no se ve reflejado en su empleo.

Palabras clave: turismo rural, empleo, desarrollo, provincias. 


\title{
Impact of the Rural Tourism on the Employment in Spain: An Approach at a Province Extent
}

\begin{abstract}
:
Rural tourism is an activity with growing importance in the rural environment, as it helps to complement the agricultural income and, all the more, to mitigate the depopulation in the rural areas. Rural tourism should be understood both as an economic option for the rural areas and as a strategic drive for the territorial development that, in turn, helps to develop those rural areas with poor economic and social conditions. Therefore, rural tourism is studied as an economic alternative for both the reinvigoration and development of rural areas, by analyzing how this activity impacts the employment in these areas. Variables providing information on three different thematic lines are analyzed: economy, demographics and rural tourism. To carry out the data analysis, this work relies on factor analysis, cluster analysis, and discriminant analysis. It focuses on three factors: tourism-jobs dynamism, tourism-rural dynamism, and quality tourism vs. job-deprived provinces. Based on these three factors the Spanish provinces are then classified in three groups regarding how the rural tourism impacts the employment, which shows an uneven influence depending on the province involved. Asturias is the Spanish province where rural tourism has impacted most positively the employment. On the other hand, the provinces Cádiz, Málaga, Las Palmas and Santa Cruz de Tenerife, despite having a rural tourism driven towards the environmental quality of their protected spaces, do not report a positive impact on the employment.
\end{abstract}

Keywords: rural tourism, employment, development, provinces.

\section{Introducción}

En el periodo que va del año 2008 al 20I2, en España se han destruido 7,2 millones de puestos de trabajo y se han creado 2,4 millones, lo que indica un saldo negativo en empleos durante dicho periodo (Rocha y Aragón, 20I2). El sector turístico en España ha sido uno de los principales impulsores para la recuperación económica y el desarrollo del país. El Plan Nacional e Integral de Turismo 2012-2015, del Ministerio de Industria, Energía y Turismo (2012), muestra que España es líder mundial en turismo vacacional, pues ocupa el segundo lugar en cuanto a gasto turístico y el cuarto por número de turistas. Dicho sector presenta un comportamiento dinámico en continua expansión, incluso en periodos de crisis, y en sinergia con otros sectores, por lo que es uno de los ejes para el desarrollo económico del país (Cuñado, Gil-Aldana y Pérez, 20II; Juaneda y Riera, 20ı; Lillo-Bañuls y Casado-Díaz, 20II; Yagüe, 2002) y se lo considera, además, fundamental para el desarrollo de determinadas regiones (Ghaderi y Henderson, 20I2; Panyik, Costa y Rátz, 20II; Pérez y Zizumbo, 20I4).

El año 2017 fue declarado como el año internacional del turismo sostenible por la Organización Mundial del Turismo (OMT), en el contexto de la Agenda 2030 y de los objetivos de desarrollo sostenible. El objetivo es sensibilizar y convencer, tanto a los responsables políticos como al público en general, de las ventajas del turismo sostenible frente al simple crecimiento económico, así como fomentar la colaboración entre todos los grupos de interés, con el fin de cambiar las políticas, las prácticas de empresa y el comportamiento de los consumidores.

El turismo tiene una gran incidencia en los recursos socioculturales y ambientales de los lugares de destino (Deery, Jago y Fredline, 20I2), por lo que es de gran importancia la consideración de estos factores en la propia gestión turística, y el turismo rural ofrece una solución alternativa al tratarse de un turismo sostenible, al no requerir grandes volúmenes de visitantes y al no necesitar un desarrollo extenso de infraestructuras (Simao y Partidario, 20I2). Además, los turistas valoran cada vez más la sostenibilidad medioambiental (Alonso-Almedia y CeleminPedroche, 2016). 
El turismo rural nace como alternativa al turismo tradicional, masivo o estandarizado (Varisco, 2016). Su característica fundamental es que se desarrolla en áreas rurales, por lo que se sustenta en principios ambientales, culturales, sociales y económicos y no posee un carácter tan marcadamente estacional como el turismo tradicional. El turista rural busca en la naturaleza un entorno saludable (Pitarch, Buciega y Esparcia, 2004), y las zonas rurales ofrecen un gran potencial turístico, ya que poseen los paisajes naturales, la cultura, la tradición y la gastronomía y, además, ofrecen actividades al aire libre (Navarro, 2015). Además, el turismo en estas zonas se convierte en una alternativa para la diversificación y la reconversión de industrias tradicionales (Zou, Huang y Ding, 2012) y permite que estas zonas puedan integrar sus actividades cotidianas con el turismo, desarrollando un paquete turístico que pueda responder a la necesidad de nuevas experiencias (Kastenholz, Carneiro, Marques y Lima, 20I2), ya que este tipo de turistas está interesado en la cultura y las tradiciones locales, lo cual permite crear una interacción entre ellos y los residentes locales (Bell y Arranz, 20II).

En las últimas décadas, el turismo rural se ha convertido en una de las principales actividades económicas en la mayoría de las zonas rurales de España. Un aspecto decisivo en este desarrollo han sido los cambios en los patrones de comportamiento de los turistas con respecto a sus motivaciones y preferencias en los viajes (Aguiló y Juaneda, 2000; Hummelbrunner y Miglbauer, 1994; Yagüe, 2002). Este auge también se ha visto favorecido por un incremento de la oferta disponible. Las grandes inversiones, financiadas fundamentalmente mediante fondos europeos, han generado la aparición en el mercado de una oferta de alojamiento amplia y heterogénea (Secretaría de Estado de Comercio, Turismo y de la Pequeña y Mediana Empresa y Ministerio de Economía, 2000).

Schejtman y Berdegué (2004) consideran la siguiente definición de desarrollo territorial rural: "Un proceso de transformación productiva e institucional en un espacio rural determinado, cuyo fin es reducir la pobreza rural” (p. 4). Esta definición refleja el vínculo de los sectores económico, institucional, social y ambiental, pues contribuye al desarrollo de la zona en la que se implanta. El turismo rural es una alternativa económica importante para las zonas rurales y un eje estratégico para el desarrollo territorial rural (Cánoves, Villarino y Herrera, 2006), que permite promover el desarrollo de algunas áreas rurales económica y socialmente deprimidas (Dernoi, I99I; Fleischer y Felsenstein, 2000; Valdés y del Valle, 2003; Yagüe, 2002). El turismo como herramienta de crecimiento rural debe adaptarse a los mecanismos de mercado actuales, que se vuelven extremadamente competitivos y que están dominados por las técnicas de comunicación y promoción (Gannon, 1994).

\section{Evolución del turismo rural en España}

En España, el medio rural ha visto cómo, con el paso del tiempo, ha perdido relevancia en la sociedad moderna española. Esta es una circunstancia común a otros países, lo que supone un fuerte coste social, pues provoca una redistribución de la población española, que se concentra mayoritariamente en zonas urbanas y las convierte en centros económicos y sociales (Ministerio de Agricultura, Pesca y Alimentación, 1992). Especial importancia ha tenido en los pequeños municipios el proceso conocido como de terciarización del medio rural, que consiste en el traslado de mano de obra del sector agrícola hacia el sector de servicios.

El modelo de turismo rural actual tiene un corto desarrollo de unos 30 años y ha experimentado un repunte muy importante en los últimos is (figura I). 


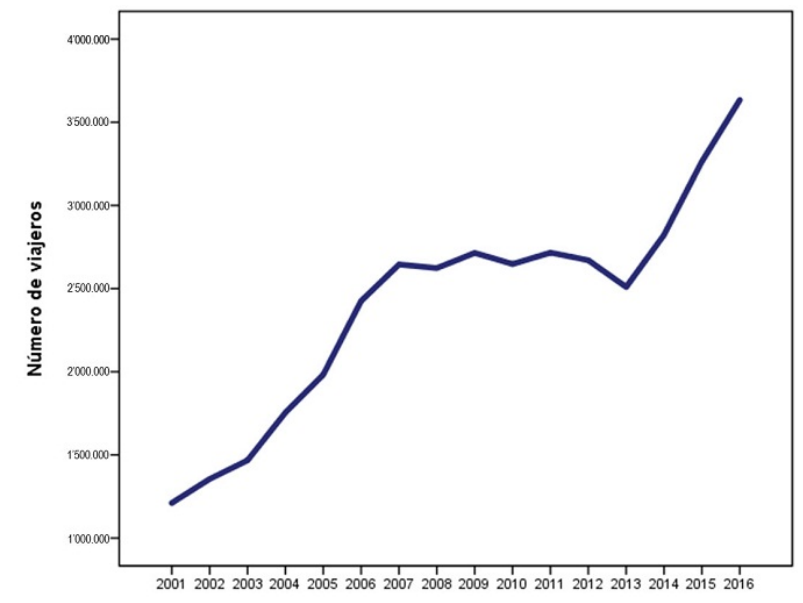

FIGURA 1

Evolución del nÚmero de turistas RuRALES En EsPaña, años 2001-2016

Fuente: elaboración propia con base en datos del Instituto Nacional de Estadística (INE)

Tiene su origen en el modelo de alojamiento en casas rurales existente en España desde la década de los 60. Su nacimiento se produjo con el programa Casas de Labranza, que puso en marcha la Agencia de Extensión Agraria, dependiente del Ministerio de Agricultura y del Ministerio de Información y Turismo, y existió una serie de factores que han ayudado a este auge. El primero de ellos es que el turismo rural se ha presentado como solución para tratar de paliar la despoblación creciente que sufren las zonas rurales. De hecho, en la década de los 90, el turismo rural contribuyó de forma importante a aminorar la migración de las zonas rurales (Paniagua, 2002) y a crear beneficios gracias a la transformación económica de las zonas en las que se implantó. Otro factor importante es la implantación de programas institucionales de desarrollo rural (Leader I, Leader II, Leader Plus, Proder) que establecen medidas de apoyo técnico al desarrollo rural, formación profesional, ayudas a la contratación, ayudas a pequeñas empresas, artesanía y servicios locales y comercialización de la producción agraria, forestal y pesquera local. El turismo rural ha tenido como principal estrategia la de conservar el patrimonio familiar y remediar la disminución de rentas derivadas de la agricultura, lo que permite la diversificación de las economías rurales. También cabe considerar la preocupación social por la protección medioambiental de las zonas rurales, posiblemente como reacción al turismo de masas que se está produciendo en España.

En España se diferencian tres fases en las que se puede analizar la evolución del turismo rural. La primera fase se sitúa en la década de los años 70 , cuando el turismo rural se basaba casi en su totalidad en el alojamiento en casas en las zonas rurales y en el arrendamiento de habitaciones, con el objetivo de proporcionar una renta adicional a la economía familiar a través del alojamiento rural (programa Casas de Labranza). Fundamentalmente, fueron las mujeres las que contribuyeron a diversificar y promocionar las actividades (Cánoves y Villarino, 1997, 2000). El turismo era de tipo tradicional y se centraba en la existencia de segundas residencias y en el uso de viviendas familiares (Soret, 1999). La segunda fase se puede situar en el periodo que va de 1980 a 1995, cuando las comunidades autónomas adquieren las competencias de la planificación turística; es por ello que durante este periodo se produce un desarrollo del turismo rural de forma desigual dependiendo de la comunidad autónoma, por lo que se favorecía una oferta turística rural diversa en cada comunidad. Los objetivos básicos que se marcan en este periodo son: a) evitar el deterioro del patrimonio arquitectónico, y para ello se hace especial hincapié en la recuperación de viviendas tradicionales con uso turístico; b) revitalizar el sector industrial complementario a la actividad agrícola y proporcionar una renta complementaria a la agricultura; c) paliar la despoblación en las zonas rurales mediante la creación de empleo, y d) revalorizar los espacios culturales, ambientales y sociales de las zonas rurales. En resumen, esta fase se caracteriza por el desarrollo de actividades alternativas al alojamiento en casas rurales y por la diversidad e integración de nuevas actividades en estas.

La última fase de la evolución del turismo rural se sitúa en el periodo actual, en el que el turismo rural en España está plenamente consolidado, es valorado por los usuarios y está extendido por todo el territorio nacional y en el 
que las comunidades autónomas le apuestan a la promoción de un producto de calidad como complemento a otras formas de turismo.

\section{Turismo rural y empleo}

El inicio, en 2008, de la crisis económica y financiera española generó preocupación en las instituciones por la creación de empleo. Se han elaborado normativas, a nivel de España y de la Comunidad Económica Europea, para las zonas rurales en las que se hace hincapié en la creación de empleo (Reglamento [CE] 1698/2005, Ley 45 de 2007). En España, estas normativas han servido para determinar los principales problemas de las zonas rurales, como son la despoblación o el envejecimiento de su población, y el planteamiento de acciones para mitigarlos, entre las que está la creación de empleo.

Para realizar un análisis de la evolución del empleo en España, se analiza la variable población ocupada, extraída de la Encuesta de Población Activa (EPA), proporcionada por el INE.

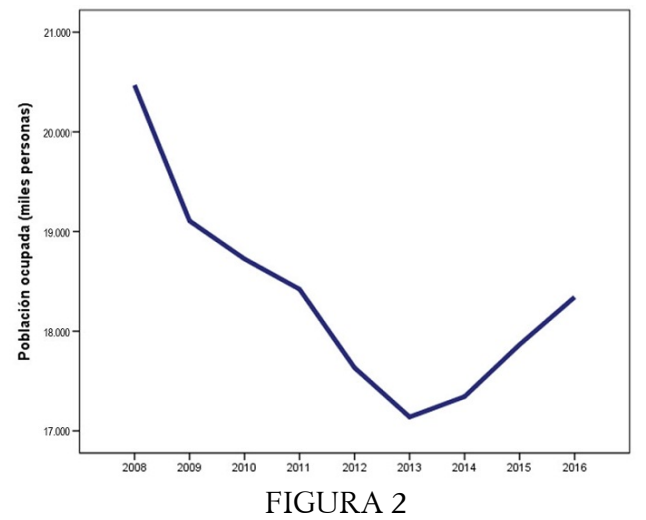

Evolución de la POBlación OCUPAda EN España, años 2008-2016

FuENTE: ELABORACión PROPIA CON BASE EN DATOS DEL INE

El comportamiento de la población ocupada en España está claramente marcado por la crisis económica, pues, a partir de 2008 , la población ocupada sufrió un gran desplome, aunque desde 2013 se observa una lenta, pero constante, recuperación en el empleo (figura 2).

$\mathrm{Al}$ igual que el resto de sectores, el sector agrario también se ha visto inmerso en una tendencia de pérdida de empleo, puesto que su población ocupada ha disminuido en la última década. Escudero (1986) explica la disminución de población ocupada en la agricultura por el abandono de las labores agrarias por parte de los agricultores, debido a la jubilación o al cambio de sector de actividad. Sin embargo, Garrido y González (1992) indican, como principal causa, la disminución en el número de explotaciones y la extensión de la mecanización del sector, lo que incide directamente en el personal empleado, aunque este contexto en el sector agrícola no es coyuntural, sino estructural, ya que esta situación se sufre desde la década de los años 60.

El sector agrícola ha vivido varias reformas en la política agraria (ayuda única por explotación, cumplimiento de normas medioambientales, reducción de presupuestos, etc.), lo que ha provocado una pérdida importante de renta y un abandono de explotaciones que inciden directamente en la pérdida de empleo en el sector agrario. Todo ello plantea un futuro algo incierto en el sector agrícola.

Una alternativa económica a esta problemática es el turismo rural, pues es relevante tanto en términos de renta como en la creación de puestos de trabajo (Jiménez, 2000; Plaza y Araque, 2000), incluso suponiendo que estos empleos tendrán características semejantes a las del empleo turístico, en el que se destaca la insuficiente 
profesionalización (Jiménez, 2000). Es un sector que incluso considera a algunos grupos tradicionalmente marginados por el mercado laboral, como son las mujeres, los jóvenes y los adultos mayores (Pérez, 2010).

El potencial del sector es un hecho, pues el número de personas empleadas en turismo rural se ha multiplicado por tres durante los últimos is años (figura 3), con una evolución constante y creciente de la población ocupada en el sector, incluso durante el grave periodo de crisis económica y financiera que ha afectado a España.

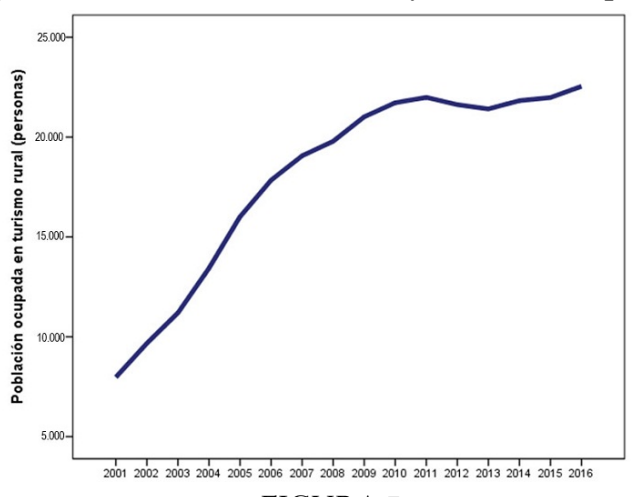

FIGURA 3

Evolución de la población OCUPADA En tURismo RURAl En EsPaña, años 2001-2016

Fuente: ELABORACión PROPIA CON BASE EN DATOS DEL INE

El turismo rural es una alternativa económica importante para las zonas rurales y un eje estratégico para el desarrollo territorial rural (Cánoves et al., 2006) que permite promover el desarrollo de algunas áreas rurales económica y socialmente deprimidas (Dernoi, 1991; Fleischer y Felsenstein, 2000; Valdés y del Valle, 2003; Yagüe, 2002). Algunos aspectos a considerar para valorar el impacto del turismo en las zonas rurales son la diversificación, el empleo, la consideración de las mujeres y los jóvenes, la revalorización del patrimonio cultural y ambiental, el incremento del producto interior bruto (PIB), el progreso comercial y productivo y el fomento de la cooperación (Barrera, 2007).

Dada la importancia del empleo como factor para valorar el impacto turístico, este trabajo se centra en el análisis de dicha variable y se plantea como principal objetivo la consideración del turismo rural como alternativa económica de revitalización en el entorno rural, por medio del análisis, fundamentalmente, del impacto del sector en términos laborales. Este análisis permitirá construir perfiles provinciales en los que se pondrá de manifiesto y se recogerán las diferencias en cuanto a aspectos de empleo y de turismo rural en las distintas provincias españolas.

\section{Metodología}

Los datos utilizados en el estudio se han obtenido de estadísticas oficiales publicadas por el Instituto Nacional de Estadística (INE) y el Instituto Geográfico Nacional de España para el año 2016.

La unidad de análisis es la provincia, entendiéndola como una demarcación administrativa española. En la actualidad, hay un total de 52 provincias, que comprenden la totalidad del territorio español. Cada provincia está dividida en un número variable de municipios y su gobierno y administración están atribuidos constitucionalmente a las diputaciones provinciales.

Las variables utilizadas en la investigación están condicionadas a la información que se encuentra en las bases de datos oficiales. Las variables seleccionadas a nivel provincial identifican tres bloques temáticos: economía, demografía y turismo rural. 
La matriz de datos está formada por 17 variables recogidas para las 50 provincias españolas (se excluyen las provincias de Ceuta y Melilla del análisis, por falta de información en muchas de las variables estudiadas). A continuación, se detallan las variables que conforman cada uno de los tres bloques temáticos.

El primero, denominado economía, recoge 5 variables importantes para caracterizarlo (entre paréntesis se muestran las unidades de medida de cada variable o indicador): tasa media de actividad (\%), tasa media de paro (\%), tasa media de empleo (\%), personal empleado en turismo rural (número de personas) y PIB per cápita (euros).

El segundo conjunto de variables, definido como demografía, recoge 4 variables: población (número de personas), densidad (habitantes $/ \mathrm{km}^{2}$ ), espacios protegidos (número) y hectáreas de espacios protegidos (hectáreas).

Por último, el bloque temático de variables al que referenciamos como turismo rural incluye 8 variables: viajeros (número de personas), pernoctaciones (número), estancia media (días), establecimientos abiertos (número), plazas ofertadas (número), grado de ocupación por plaza (\%), grado de ocupación por plaza en fin de semana (\%) y grado de ocupación por habitaciones (\%).

Las variables están recogidas en una escala muy dispar, lo que hace necesaria su estandarización (a cada variable se le resta su media y el resultado se divide por su desviación típica), que permitirá realizar el análisis en términos relativos y comparables.

Para el análisis de datos, se aplicarán técnicas estadísticas de análisis multivariante, en concreto, análisis factorial, análisis clúster y análisis discriminante. Para poder aplicar estas técnicas, se han comprobado las siguientes hipótesis: normalidad multivariante, homocedasticidad multivariable e independencia de las distribuciones marginales.

Para el análisis de la relevancia del turismo rural en la economía social y laboral de las provincias españolas, se aplicará el análisis factorial. Esta técnica persigue identificar los factores que permitan explicar las relaciones dentro de las variables, de forma que expresen la realidad del turismo rural social y laboralmente en las provincias españolas. De esta forma, se resume la información de las variables originales mediante combinaciones de estas, extrayéndose los factores buscados. Esto permite obtener un menor número de variables no observadas o latentes (lo que se conoce como factores), que expresan de una manera sencilla el comportamiento de un grupo de individuos sobre los que se ha estudiado un conjunto de variables de partida.

Para obtener los factores, se utilizará el método de extracción de componentes principales (Morrison, 1987), dado que el análisis se realizará desde una perspectiva exploratoria y no inferencial. El criterio que permitirá la selección de factores será la elección del autovalor mayor de la unidad (Kaiser, 1960). El método de rotación ortogonal varimax facilitará la interpretación de los factores que se obtengan.

Una vez obtenidos los factores mediante el análisis factorial, estos permitirán identificar conjuntos de provincias con características sociales, laborales y de turismo rural similares, mediante la aplicación del análisis clúster, también conocido como análisis de conglomerados. Esta técnica estadística de clasificación permite agrupar individuos con características similares, y así identifica conjuntos de casos semejantes (Hair, Anderson, Tatham y Black, 2000). Para la aplicación del análisis clúster, se usará la distancia euclídea al cuadrado como medida de similitud entre los individuos (en nuestro caso, las provincias), midiendo lo cerca o lejos que se encuentran los valores de las variables. Para formar los clústeres, se dispone de dos métodos, los jerárquicos y los no jerárquicos. Los primeros contemplan todas las posibles agrupaciones, que van desde un solo clúster, en el que estarán todos los individuos, hasta tantos clústeres como individuos haya. Los métodos no jerárquicos se caracterizan por llevar a cabo agrupaciones de individuos en un número fijado de clústeres. En nuestro análisis, en primer lugar, se aplicará un procedimiento jerárquico con el que se determinará el número de clústeres, k, más adecuado. Posteriormente, se aplicará el método no jerárquico de k-medias.

Por último, el análisis discriminante permitirá validar la bondad de los factores extraídos con el análisis factorial y la agrupación realizada con el análisis clúster. Este análisis tomará como punto de partida un grupo de elementos que pertenecerán a distintos conjuntos previamente fijados y examinará la información referente a distintas 
variables, con un doble objetivo: un fin explicativo, que tratará de establecer la contribución de cada variable a la correcta clasificación de los elementos, y un fin predictivo, con el que se tratará de determinar el grupo en el que se clasificará un nuevo elemento, lo que permite analizar la capacidad de discriminación de los factores fijados.

El tratamiento estadístico de los datos se ha realizado mediante el programa de IBM SPSS Statistics v22.0.0.

\section{Resultados}

Para poder establecer la influencia del turismo rural en aspectos relacionados con el empleo del sector y, con ello, extraer un perfil de su impacto a nivel provincial, se estructurarán los resultados en tres cuestiones: análisis factorial, análisis clúster y análisis discriminante.

\section{Análisis factorial}

Para el análisis de la influencia del turismo rural en la realidad social y laboral de las provincias españolas se utilizan las i7 variables ya indicadas.

Para comprobar si el modelo factorial es adecuado y aplicable, se analiza la matriz de correlaciones asociada a las variables, y para ello se aplica la prueba de esfericidad de Bartlett, que permite contrastar si dicha matriz es una matriz identidad. Cuanto mayor sea el estadístico de Bartlett $(1676,803)$ y menor valor tenga el nivel de significación (0,0000), menos probable es que la matriz de correlaciones sea una matriz identidad, y así resultaría correcta la aplicación del análisis factorial.

El criterio fijado para la elección de autovalores seleccionará aquellos que sean los mayores de la unidad; esto permite extraer tres factores que explican el 69,II4\% de la varianza total (tabla I). Este porcentaje es aceptable dado que, en estudios relacionados con disciplinas de ciencias sociales, el límite de aceptación inferior está fijado en aproximadamente un 60\% (Hair et al., 2000). Cada uno de los tres factores extraídos explica el 30,438\%, el 21,454\% y el $17,221 \%$ de la varianza total (tabla I).

TABLA 1

FACTORES EXTRAÍDOS Y VARIANZA EXPLICADA POR EL MODELO

\begin{tabular}{lccr}
\hline & Autovalor & $\begin{array}{c}\text { Porcentaje } \\
\text { de varianza }\end{array}$ & $\begin{array}{c}\text { Porcentaje } \\
\text { de varianza } \\
\text { acumulado }\end{array}$ \\
\hline Factor 1: Dinamismo turístico-laboral & 5,175 & 30,438 & 30,438 \\
\hline Factor 2: Dinamismo turístico-rural & 3,647 & 21,454 & 51,893 \\
\hline $\begin{array}{l}\text { Factor 3: Turismo de calidad vs. } \\
\text { provincias "deprimidas" laboralmente }\end{array}$ & 2,928 & 17,221 & 69,114 \\
\hline
\end{tabular}

Fuente: elaboración propia 
TABLA 2

Pesos FACTORiales de LA MATRiz FACTORial RotAdA

\begin{tabular}{lccc}
\hline Variables & Factor 1 & Factor 2 & Factor 3 \\
\hline Viajeros & 0,883 & 0,034 & $-0,264$ \\
\hline Pernoctaciones & 0,941 & 0,008 & $-0,142$ \\
\hline Estancia media & 0,106 & 0,492 & 0,708 \\
\hline Establecimientos abiertos & 0,931 & $-0,031$ & 0,111 \\
\hline Plazas ofertadas & 0,989 & 0,010 & 0,020 \\
\hline Grado de ocupación por plaza & 0,043 & 0,835 & 0,168 \\
\hline Grado de ocupación por plaza en fin & 0,069 & 0,769 & $-0,250$ \\
de semana & & & \\
\hline Grado de ocupación por habitaciones & 0,051 & 0,890 & 0,062 \\
\hline Personal empleado en turismo rural & 0,868 & 0,255 & 0,131 \\
\hline Tasa media de actividad & $-0,090$ & 0,717 & $-0,009$ \\
\hline Tasa media de paro & $-0,292$ & $-0,263$ & 0,825 \\
\hline Tasa media de empleo & 0,164 & 0,634 & $-0,617$ \\
\hline Población & 0,046 & 0,451 & $-0,044$ \\
\hline Densidad & 0,044 & 0,454 & $-0,038$ \\
\hline Espacios protegidos & 0,133 & 0,416 & 0,584 \\
\hline Hectáreas de espacios protegidos & 0,043 & $-0,049$ & 0,701 \\
\hline PIB per cápita & 0,098 & 0,518 & $-0,687$ \\
\hline & & &
\end{tabular}

Fuente: elaboración propia

La tabla 2 proporciona la matriz factorial rotada, que recoge los coeficientes de correlación lineal entre los factores extraídos y las variables originales y refleja así el peso o la importancia de cada variable en cada factor, de forma que los factores con un peso factorial más alto en términos absolutos muestran una relación más fuerte con las variables. El análisis establece una relación fuerte del primer factor con 5 de las i7 variables estudiadas (viajeros, pernoctaciones, establecimientos abiertos, plazas ofertadas y personal empleado en turismo rural). Esta relación se determina por los valores de las correlaciones positivas de las variables indicadas. Las variables de mayor peso en el factor indican que valores elevados (o bajos) del factor están relacionados con provincias con alto (o bajo) número de viajeros, de pernoctaciones, de establecimientos abiertos, de plazas ofertadas y de personal empleado en el sector del turismo rural. Por el contrario, el primer factor está correlacionado negativamente con las variables de tasa media de actividad y tasa media de paro. Esto significa que valores altos (o bajos) del factor están relacionados con provincias con baja (o alta) tasa media de actividad y baja (o alta) tasa media de paro (tabla 2).

Las variables que caracterizan al primer factor recogen aspectos relacionados con el turismo rural y el empleo del sector, lo que permite etiquetar el factor como dinamismo turístico-laboral. 
Las provincias que obtienen puntuaciones más elevadas en el primer factor son las que destacan en cuanto al dinamismo turístico-laboral. Estas provincias, por orden de relevancia o mayor puntuación en este factor, son las siguientes: Asturias (4,43), Cantabria (I,73), Girona (I,44), Ávila $(\mathrm{I}, 38)$, Navarra $(\mathrm{I}, 35)$ y Cáceres $(\mathrm{I}, 2 \mathrm{I})$.

Las provincias que integran el segundo factor tienen una relación fuerte y positiva con 5 de las i7 variables (grado de ocupación por plaza, grado de ocupación por plaza en fin de semana, grado de ocupación por habitaciones, tasa media de actividad y tasa media de empleo). Esto indica que valores altos (o bajos) del segundo factor están asociados a provincias con altos (o bajos) grados de ocupación por plaza, de ocupación por plaza en fin de semana, de ocupación por habitaciones y altas tasas medias de actividad y de empleo (tabla 2). La relación del segundo factor es negativa con las variables de tasa media de paro, establecimientos abiertos y hectáreas de espacios protegidos, aunque la relación con las dos últimas variables es débil. Estas relaciones indican que valores altos (o bajos) del segundo factor están asociados a provincias con baja (o alta) tasa media de paro, de establecimientos abiertos y de hectáreas de espacios protegidos. Estos resultados permiten etiquetar este factor como dinamismo turístico-rural.

Las provincias que obtienen mayores puntuaciones en el factor dinamismo turístico-rural y que, por tanto, destacan en importancia en dicho factor son: Illes Balears (3,27), Madrid (2,02), Barcelona (1,82), Guipúzcoa ( 1,63$)$ y Badajoz (I,II).

El tercer factor se ha etiquetado como turismo de calidad versus provincias “deprimidas" laboralmente, pues los municipios que lo integran destacan por el tiempo de estancia media, las hectáreas de espacios protegidos y la tasa media de paro (tabla 2). La correlación de este factor es positiva con las variables antes indicadas, es decir, valores altos (o bajos) del factor están asociados a provincias con altos (o bajos) valores de estancia media, de hectáreas de espacios protegidos y de tasa media de paro. Además, está correlacionado negativamente con las variables de tasa media de empleo, PIB per cápita, número de viajeros, pernoctaciones y grado de ocupación por plaza en fin de semana (densidad, población y tasa media de actividad están relacionadas negativamente pero de forma muy débil con el tercer factor). Esta correlación indica que valores altos (o bajos) del factor están relacionados con provincias con baja (o alta) tasa media de empleo y bajos (o altos) valores de PIB per cápita, de viajeros, de pernoctaciones y de grado de ocupación por plaza en fin de semana. Las provincias más relevantes por su alta puntuación en el tercer factor son Santa Cruz de Tenerife (2,67), Cádiz (2,16), Málaga (2,I4), Las Palmas (I,74), Jaén (I,47), Córdoba (I,2I) y Huelva (I,20).

\section{Análisis clúster}

La siguiente etapa del análisis es la aplicación del análisis clúster. El objetivo de esta técnica es obtener provincias con características similares respecto a aspectos de turismo rural, laboral y social para los factores indicados.

El método seleccionado para la obtención de los clústeres es el no jerárquico, y se aplicó en las puntuaciones obtenidas en las distintas provincias para los tres factores extraídos, con la necesidad de conocer a priori el número de clústeres a definir. Hair, Anderson y Tatham (1987) explican los beneficios de los métodos jerárquicos, pero reconocen las ventajas y la mayor extensión de los métodos no jerárquicos. Entre las ventajas que indican, se encuentra el hecho de que permiten trabajar con un número muy elevado de individuos y variables. Se realizará en primer lugar un análisis clúster mediante procedimientos jerárquicos, lo que permitirá determinar el número de clústeres más adecuado, así como la configuración de estos, que servirá como punto de partida.

Tras realizar distintas pruebas con los métodos jerárquico y no jerárquico, el análisis de las variaciones que se producen en la varianza residual, al considerar distintos números de grupos, lleva a fijar cuatro clústeres como número óptimo.

La tabla 3 muestra las puntuaciones medias de los clústeres en los diferentes factores y pone de manifiesto la heterogeneidad de las provincias españolas. 
TABLA 3

Puntuaciones medias en los Clústeres

\begin{tabular}{lcccc}
\hline & \multicolumn{3}{c}{ Clúster } \\
\cline { 2 - 5 } & 1 & 2 & 3 & 4 \\
\hline Factor 1: Dinamismo turístico-laboral & $-0,23756$ & 4,43336 & 0,34126 & $-0,31695$ \\
\hline Factor 2: Dinamismo turístico-rural & $-0,54617$ & $-1,04344$ & 1,15391 & 1,06962 \\
\hline $\begin{array}{l}\text { Factor 3: Turismo de calidad vs. } \\
\text { provincias "deprimidas" laboralmente }\end{array}$ & 0,00940 & 1,04011 & $-0,77497$ & 2,18343 \\
\hline
\end{tabular}

Fuente: elaboración propia

La tabla 4 recoge las provincias que forman parte de cada uno de los clústeres.

TABLA 4

Pertenencia de las provincias a los Clústeres

\begin{tabular}{cl}
\hline Clúster & Provincias \\
\hline 1 & $\begin{array}{l}\text { 32 provincias: A. Coruña, Albacete, Alicante, Almería, Ávila, Badajoz, Cáceres, } \\
\text { Castellón, Ciudad Real, Córdoba, Cuenca, Granada, Guadalajara, Huelva, Huesca, } \\
\text { Jaén, León, Lugo, Murcia, Ourense, Palencia, Pontevedra, Salamanca, Sevilla, } \\
\text { Soria, Tarragona, Teruel, Toledo, Valencia, Valladolid, Zamora y Zaragoza }\end{array}$ \\
& Una provincia: Asturias \\
\hline 3 & $\begin{array}{l}\text { 13 provincias: Álava, Illes Balears, Barcelona, Bizkaia, Burgos, Cantabria, } \\
\text { Guipúzcoa, Girona, Lleida, Madrid, Navarra, La Rioja y Segovia }\end{array}$ \\
\hline 4 & 4 provincias: Cádiz, Málaga, Las Palmas, Santa Cruz de Tenerife
\end{tabular}

Fuente: elaboración propia

La figura 4 representa las puntuaciones medias de cada clúster en los diferentes factores. De esta se desprende que el clúster 2 registra la puntuación media más elevada respecto al primer factor, que representa el dinamismo turístico-laboral (tabla 3 y figura 4); es decir, la provincia de Asturias es la más destacada en cuanto al factor dinamismo turístico-laboral, por lo que en esta provincia el turismo rural tiene un impacto bastante notable sobre el empleo. El clúster 4 recoge la mayor puntuación media respecto al tercer factor, por lo que las provincias que integran el clúster 4 representan un turismo de calidad vs provincias “deprimidas” laboralmente (Cádiz, Málaga, Las Palmas y Santa Cruz de Tenerife). El clúster 3 representa aquellas provincias (Álava, Illes Balears, Barcelona, Bizkaia, Burgos, Cantabria, Guipúzcoa, Girona, Lleida, Madrid, Navarra, La Rioja y Segovia) que destacan en dinamismo turístico-rural, es decir, que destacan por el grado de ocupación turística rural y presentan tasas de actividad y empleo elevadas, aunque esto no supone un impacto directo en el empleo asociado al turismo rural. El clúster I no destaca especialmente en cuanto a puntuación media en ninguno de los factores; las provincias que pertenecen a este clúster están en la puntuación media de los factores i y 3, mientras que están un poco por debajo de la media del factor 2. 


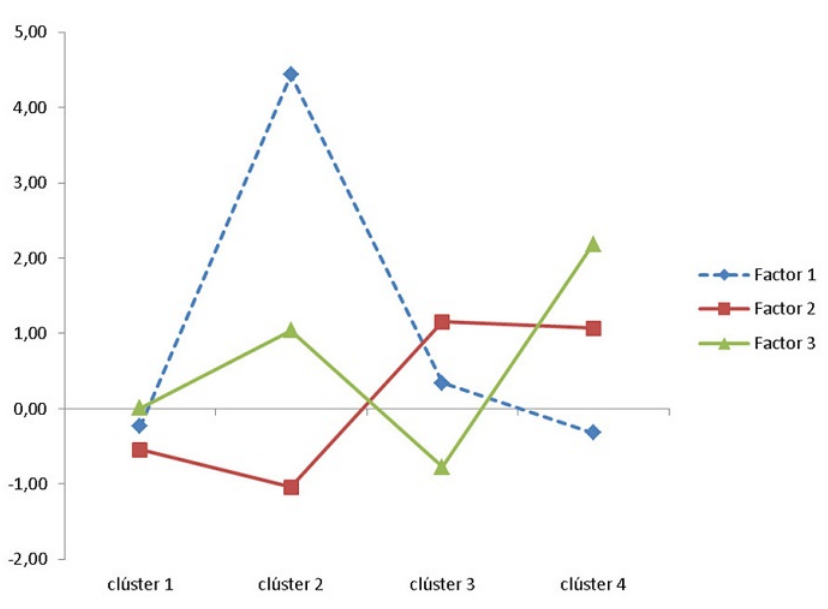

FIGURA 4

Puntuaciones medias de los Clústeres en los Factores

FUENTE: ELABORACIÓN PROPIA

Las puntuaciones medias de los clústeres (tabla 3 y figura 4 ) permiten clasificar las provincias en cuanto al impacto del turismo rural en el empleo en tres grupos. En el primero están las provincias que presentan una situación turística y laboral buena; estas provincias son las que obtienen mayor puntuación media en cuanto al factor dinamismo turístico-laboral (factor I), integrado por la provincia de Asturias, o incluso se puede ver como el clúster que menor puntuación obtiene en el factor 3. Por otra parte, están las provincias que presentan una situación turística y laboral media; a este grupo pertenecerían las 32 provincias que integran el clúster i y las iz provincias pertenecientes al clúster 3 (tabla 4 ), aunque hay que indicar que las provincias integrantes del clúster 3 tienen una situación laboral algo mejor que las que pertenecen al clúster I, pues así lo indican las puntuaciones medias de los clústeres en los factores (tabla 3). Por último, se pueden clasificar las provincias que poseen una situación turística y laboral mala, clúster integrado por las provincias de Cádiz, Málaga, Las Palmas y Santa Cruz de Tenerife (figura 4).

En la figura 5 se representan las provincias españolas que integran cada uno de los 4 clústeres construidos. El clúster i es el más numeroso en cuanto a provincias que lo componen, pues está integrado por 32; en este se recoge prácticamente la totalidad de provincias españolas, sin distinción entre mitad sur o norte: recordemos que estas provincias presentan una situación turística y laboral media. El clúster 3 está formado fundamentalmente por provincias de la mitad más septentrional de España; estas zonas tienen un fuerte arraigo del turismo rural, y además, este tiene un impacto medio sobre el empleo en dichas provincias. El clúster 2 está conformado por la provincia de Asturias, que tiene una larga tradición en turismo rural y un impacto muy destacado y positivo sobre la situación laboral de la provincia. El clúster 4 integra provincias de la parte más meridional de la península ibérica (incluidas las Islas Canarias); estas presentan un turismo que busca la calidad medioambiental pero que no incide de una manera notable sobre el empleo de la zona.

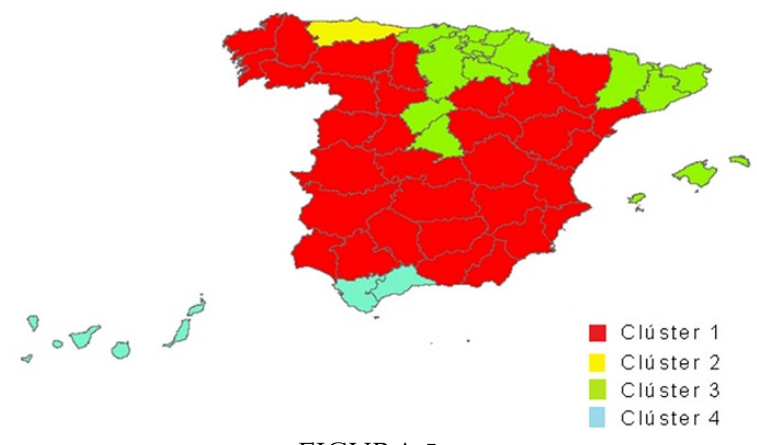

FIGURA 5

REPRESENTACIÓN DE LOS CLÚSTERES

FUENTE: ELABORACIÓN PROPIA 


\section{Validación de resultados}

El análisis discriminante permitirá validar la bondad de los resultados obtenidos, y para ello se utilizará, como variable dependiente, el clúster de pertenencia de cada provincia, y como variables independientes, los tres factores extraídos del análisis factorial. Este método exige la igualdad de las matrices de varianzas-covarianzas de los grupos. Los resultados del estadístico M de Box $(26,315)$ y el nivel de significación asociado al test $(0,090)$ confirman este requisito.

TABLA 5

Test de VAlidación DE REsultados

\begin{tabular}{lcc}
\hline & $\mathbf{C h i}^{\mathbf{2}}$ & $\begin{array}{c}\text { Nivel de } \\
\text { significación }\end{array}$ \\
\hline Factor 1: Dinamismo turístico-laboral & 114,683 & $0,000^{*}$ \\
\hline Factor 2: Dinamismo turístico-rural & 63,958 & $0,000^{*}$ \\
\hline $\begin{array}{l}\text { Factor 3: Turismo de calidadvs. provincias } \\
\text { "deprimidas" laboralmente }\end{array}$ & 27,550 & $0,000^{*}$ \\
\hline
\end{tabular}

Fuente: elaboración propia

La tabla 5 muestra el estadístico $\mathrm{Chi}^{2}$ y el nivel de significación para la validación del modelo construido. Estos resultados permiten confirmar que el análisis realizado es adecuado, dado que los factores extraídos son diferentes para los distintos clústeres. Este resultado es un indicio claro de la validez del análisis y del poder discriminante de los factores extraídos.

Además, se estudia el poder de clasificación del modelo, y se obtiene que el $98 \%$ de las provincias se clasifican correctamente (tabla 6), por lo que los resultados demuestran la alta fiabilidad del modelo construido y analizado.

TABLA 6

Predicción del Clúster de Pertenencia (porcentaje)

\begin{tabular}{ccccc}
\hline Clúster & $\mathbf{1}$ & $\mathbf{2}$ & $\mathbf{3}$ & $\mathbf{4}$ \\
\hline 1 & 100 & 0 & 0 & 0 \\
\hline 2 & 0 & 100 & 0 & 0 \\
\hline 3 & 7,7 & 0 & 92,3 & 0 \\
\hline 4 & 0 & 0 & 0 & 100 \\
\hline
\end{tabular}

Fuente: elaboración propia

Nota: El $98 \%$ de los casos agrupados originales fueron clasificados correctamente. 


\section{Discusión}

El desarrollo económico en las zonas rurales en España se sustenta sobre todo en el sector primario, aunque en los últimos años se han ido incorporando actividades distintas, entre las que se destaca el turismo, que está permitiendo que los habitantes de estas zonas puedan disponer de rentas complementarias y además está generando puestos de trabajo. De manera análoga a Swarbrooke (1996), se comprueba que este tipo de turismo está sirviendo para el desarrollo de regiones en las que existen fuertes desequilibrios socioeconómicos, lo que provoca una disminución de la dependencia respecto al sector primario.

Cánoves, Herrera y Blanco (2005) señalan un tipo de turismo rural esporádico, habitualmente en entornos periféricos y alejado de las ciudades, como sería el caso de las comunidades autónomas de Extremadura, Castilla la Mancha, Valencia y la comunidad de Murcia. Por otro lado, identifican otro tipo de turismo rural más intensivo debido fundamentalmente a los traslados de fin de semana o a los periodos vacacionales cortos, que se desarrolla en zonas cercanas a las grandes ciudades, como Cataluña y Madrid. Sin embargo, en nuestros resultados se muestra que actualmente el turismo rural es un fenómeno global en todo el territorio español, ya que tiene en todas las provincias un impacto positivo en el desarrollo de dicho entorno, dado que las variables que determinan cada uno de los factores extraídos están directamente relacionadas con el sector del turismo rural. Análogamente a Cánoves, Garay y Duro (20I2), los resultados obtenidos permiten comprobar que el turismo rural está consolidado en España y que es una de las opciones más interesantes para la elaboración de estrategias públicas de desarrollo en las zonas rurales. A ello ha contribuido el apoyo de las instituciones y organismos públicos al turismo rural, lo que sin duda ha tenido un impacto sobre el sector; sin embargo, el efecto ha sido diferente según la zona geográfica (Arias, 2013; Rico, 2005).

Nuestros resultados ponen de manifiesto la desigual capacidad del sector turístico rural para crear empleo según la provincia analizada, pues incide positivamente en el empleo en algunas provincias y no tanto en otras. Este resultado es semejante a los obtenidos por Parada y Rodríguez (2008), Plaza y Araque (2000), Jiménez (2000) y Pulido y Cárdenas (201I), en los que se demuestra que el turismo rural incide directamente sobre la creación de empleo, lo que ayudará a la redistribución de los ingresos.

Este sector tiene gran relevancia económica en las zonas rurales, pues genera un tipo de empleo que no sufre tan directamente la temporalidad como el creado por el turismo tradicional. Al igual que Ciruela (2008) y Millán, Techera y Morales (20I2), los resultados nos permiten indicar que el turismo rural permite disminuir las tasas de desempleo en las zonas rurales, dado que surge como alternativa al turismo de sol y playa, debido, en general, al cambio experimentado por la demanda de los consumidores turísticos. Esta metamorfosis de la demanda ha favorecido la posibilidad de generar empleo en el área rural a través de la actividad turística.

\section{Conclusiones}

El turismo rural tiene una proyección importante en el área rural. La creación de empleo y el incremento de las rentas pueden disminuir e incluso impedir la despoblación de las zonas rurales. El turismo es de las actividades económicas que mejor está resistiendo la crisis económica, y de ello se ha favorecido el turismo rural, que ha creado empleo en periodos en los que lo habitual era la pérdida de este.

Se comprueba que el impacto provocado por el turismo rural sobre el empleo es diferente dependiendo de la provincia analizada. Esta influencia puede explicarse por factores como el nivel de desarrollo turístico de cada zona, 
su extensión, así como la presencia de parajes con encanto ambiental, pues son factores que inciden directamente sobre el desarrollo del turismo rural en ciertas provincias.

Asturias, con poco turismo de sol y playa, no es el destino turístico clásico, sin embargo, dispone de un variado patrimonio arquitectónico, natural y paisajístico y ofrece una extensa oferta patrimonial, gastronómica y cultural, por lo que se destaca especialmente en aspectos relacionados con el número de viajeros, de pernoctaciones, de establecimientos abiertos, de plazas ofertadas y de personal empleado en turismo rural, que inciden de manera muy notable sobre el empleo de la provincia. Asturias es la provincia española en la que el turismo rural tiene un mayor impacto positivo sobre el empleo del sector.

El análisis identifica que las variables de grado de ocupación por plaza, por plaza en fin de semana, ocupación por habitación, tasa de actividad y tasa de empleo son las que caracterizan el desarrollo turístico rural en determinadas zonas, como son: Álava, Bizkaia, Guipúzcoa, Barcelona, Girona, Lleida, Burgos, Segovia, Illes Balears, Cantabria, Madrid, Navarra y La Rioja. Algunas de estas provincias son un destino turístico clásico de sol y playa en el territorio español (Illes Balears y la costa catalana) y además cuentan con un rico patrimonio natural, paisajístico y arquitectónico, por lo que también están apostando por un turismo respetuoso con la naturaleza y el entorno que se refleja de manera discreta, por el momento, sobre el empleo del sector. En contraposición, se encuentran las provincias de Cádiz, Málaga, Las Palmas y Santa Cruz de Tenerife, caracterizadas por un bajo impacto en el empleo del sector, aunque hay que indicar que el turismo de estas zonas busca parajes con encanto y protección ambiental y destaca por los días de estancia media; sin embargo, esto no se refleja de forma importante en el empleo de dichas provincias. Este resultado se puede entender en parte debido a que estas provincias tradicionalmente son zonas con gran importancia en turismo de litoral, y, a pesar de disponer de entornos protegidos muy importantes a nivel nacional (parque natural de Doñana, parque natural de la Sierra de Grazalema, parque nacional del Teide, etc.), aún el turismo tradicional de sol y playa le gana la partida del empleo al turismo rural.

El turismo rural no es la solución completa para el problema económico y poblacional que sufren las zonas rurales, pero sí puede servir como plataforma para dar a conocer la riqueza de estos entornos, frecuentemente desconocidos, pero que presentan un poderoso potencial de cara al impulso de acciones paralelas y complementarias al sector del turismo rural.

Para que el turismo rural tenga un efecto más positivo sobre el empleo y pueda ayudar a dinamizar la economía del entorno, es conveniente que las administraciones públicas tomen medidas que contribuyan a mejorar servicios básicos de las áreas rurales, como son la educación, la sanidad y las infraestructuras de comunicación, lo que sin duda repercutirá en la calidad de vida de su población y del turista. Es importante tener un punto de vista estratégico del sector que agregue transversalmente la agricultura, el desarrollo y el turismo, sin que ello produzca una saturación del entorno rural y que logre ambientes sostenibles y genere empleo y riqueza.

Los resultados obtenidos hacen pensar en un análisis más amplio del sector que permita identificar la demanda de turismo rural, con el objetivo de conocer si el turismo que se desarrolla en determinada zona es sostenible o si mejora la calidad de vida de los habitantes e incluso de comprobar si incide sobre el nivel de ingresos de los habitantes de las zonas rurales. Ello permitirá establecer si la concepción del desarrollo rural ha cambiado, lo que favorecerá la correcta planificación en las áreas rurales.

A pesar de que los resultados muestran que el turismo rural ha tenido un fuerte crecimiento y desarrollo en el territorio español, sería interesante hacer la comparativa de este fenómeno turístico con el que ofrecen otros países europeos, especialmente de Europa occidental, donde este sector está más consolidado y tiene una mayor tradición.

\section{Referencias}

Aguiló, E., y Juaneda, C. (2000). Tourist expenditure for mass tourism markets. Annals of Tourism Research, 27(3), 624-637. https://www.doi.org/10.1016/SoI60-7383(99)00101-2 
Alonso-Almedia, M. M., y Celemin-Pedroche, M. S. (2016). Competitividad y destinos turísticos sostenibles. Esic Market Economics and Business Journal, 47(2), 29I-306. https://www.doi.org/10.7200/esicm.153.0472.3e

Arias, P. (2013). La situación del empleo en turismo rural en España. Estudios de Economía Aplicada, 3I(I), I-2I. Recuperado de https://www.revista-eea.net/documentos/31104.pdf

Barrera, E. (2007). El turismo rural: un agronegocio para el desarrollo de los territorios rurales. En C. Vieytez (ed.), Agronegocios alternativos, enfoque, importancia y bases para la generación de actividades agropecuarias no tradicionales. Buenos Aires: Hemisferio Sur.

Bell, C., y Arranz, A. (20II). El turismo y el desarrollo rural en los parques naturales: el caso del Parque Natural de Grazalema (Cádiz-Málaga). Spanish Journal of Rural Development, 2(2), I-22.

Cánoves, G., Garay, L., y Duro, J. A. (20I2). Turismo rural en España: avances y retrocesos en los últimos veinte años. Papers de Turisme, (5I), 7-2I. Recuperado de https://www.papersdeturisme.gva.es/ojs/index.php/Pap ers/article/view/I94/I6I

Cánoves, G., Herrera, L., y Blanco, A. (2005). Turismo rural en España: un análisis de la evolución en el contexto europeo. Cuadernos de Geografía, (77), 4I-58. Recuperado de https://www.uv.es/cuadernosgeo/CG77_04I_o 58.pdf

Cánoves, G., Villarino, M., y Herrera, L. (2006). Políticas públicas, turismo rural y sostenibilidad: difícil equilibrio. Boletín de la Asociación de Geógrafos Españoles, (4I), 199-217. Recuperado de https://www.dialnet.unirioj a.es/servlet/articulo?codigo=1958916

Cánoves, G., y Villarino, M. (1997). El turismo rural en Cataluña y Galicia, una alternativa o complemento de la actividad agraria familiar: las mujeres sus nuevas protagonistas. En M. Valenzuela (coord.), Los turismos de interior: el retorno de la tradición viajera (pp. 353-368). Madrid: Universidad Autónoma de Madrid, AGE, Obra Social y Cultural de Caja Castilla la Mancha.

Cánoves, G., y Villarino, M. (2000). Turismo en espacio rural en España: actrices e imaginario colectivo. Documents d'Anàlisi Geogràfica, (37), 5i-77. Recuperado de https://www.raco.cat/index.php/DocumentsA nalisi/article/view/31725/31559

Ciruela, A. M. (2008). Diversificación de la actividad agraria hacia el turismo rural: un modelo de decisión basado en sociedades cooperativas agrarias oleícolas. Ciriec: España. Revista de Economía Pública, Social y Cooperativa, (6I), 205-232. Recuperado de https://www.ciriec-revistaeconomia.es/banco/6ro9_Ciruela.pdf

Cuñado, J., Gil-Aldana, L. A., y Pérez, F. (20II). Modelling international monthly tourist in Spain. Revista de Estudios de Economía Aplicada, 29(3), 723-736. Recuperado de https://www.redalyc.org/articulo.oa?id=30I 22405004

Deery, M., Jago, L., y Fredline, L. (2012). Rethinking social impacts of tourism research: A new research agenda. Tourism Management, 33(I), 64-73. https://www.dx.doi.org/10.1016/j.tourman.20II.01.026

Dernoi, L. A. (199I). About rural and farm tourism. Tourism Recreation Research, I6(I), 3-6. https://www.doi.or g/I0.1080/0250828I.1991.110I4590

Escudero, G. (1986). Actividad, ocupación y productividad agraria en España: un análisis de la población y del empleo. Revista de Estudios Agrosociales, (137), 379-417. Recuperado de https://www.mapama.gob.es/mini sterio/pags/biblioteca/revistas/pdf_reas/r137_29.pdf

Fleischer, A., y Felsenstein, D. (2000). Support for rural tourism. Does it make a difference? Annals of Tourism Research, 27(4), 1007-1024. https://www.doi.org/10.1016/Sor60-7383(99)00126-7

Gannon, A. (1994). Rural tourism as a factor in rural community economic development for economies in transition. Journal of Sustainable Tourism, 2(I-2), 5I-59. https://www.doi.org/10.1080/09669589409510683 
Garrido, L., y González, J. (1992). El mercado de trabajo agrario: algunas cuestiones metodológicas y discusión de resultados. Revista de Estudios Regionales, (31), 45-53. Recuperado de https://www.revistaestudiosregional es.com/documentos/articulos/pdf352.pdf

Ghaderi, Z., y Henderson, J. C. (20I2). Sustainable rural tourism in Iran: A perspective from Hawraman village. Tourism Management Perspectives, 2-3, 47-54. https://www.dx.doi.org/I0.I0I6/j.tmp.20I2.03.00I

Hair, J. F., Anderson, R. E., Tatham, R. L., y Black, W. C. (2000). Análisis multivariante. Madrid: Prentice Hall Iberia.

Hair, J. F., Anderson, R. E., y Tatham, R. L. (1987). Multivariate data analysis. Nueva York: Macmillan.

Hummelbrunner, R., y Miglbauer, E. (1994). Tourism promotion and potential in peripheral areas: The Austrian case. Journal of Sustainable Tourism, 2(I-2), 4I-50. https://www.doi.org/I0.1080/09669589409510682

Jiménez, F. J. (2000). Turismo rural y empleo. En Junta de Castilla y León y Consejería de Economía y Hacienda, VII Congreso de Economía Regional de Castilla y León. Soria. Recuperado de https://www.dialnet.unirio ja.es/servlet/libro?codigo=558 118

Juaneda, C., y Riera, A. (20II). La oportunidad de la investigación en economía del turismo. Revista de Estudios de Economía Aplicada, 29(3), 711-722. Recuperado de https://www.revista-eea.net/listado.php

Kaiser, H. F. (1960). The application of electronic computers to factor analysis. Educational and Psychological Measurement, (20), I4I-15I. https://www.dx.doi.org/I0.I177/001316446002000II6

Kastenholz, E., Carneiro, M. J., Marques, C. P., y Lima, J. (2012). Understanding and managing the rural tourism experience. The case of a historical village in Portugal. Tourism Management Perspectives, 4, 207-214. http s://www.dx.doi.org/10.1016/j.tmp.2012.08.009

Lillo-Bañuls, A., y Casado-Díaz, J. M. (20II). Capital humano y turismo: rendimiento educativo, desajuste y satisfacción laboral. Revista de Estudios de Economía Aplicada, 29(3), 755-780. Recuperado de https://ww w.redalyc.org/articulo.oa?id=30122405006

Millán, M. G., Techera, J. L., y Morales, F. (2012). Rural tourism as an alternative to the development for rural areas and the creation of employment. International Journal of Humanities and Social Science, 2(20), 162-174. Recuperado de https://www.ijhssnet.com/journals/Vol_2_No_20_Special_Issue_October_20I2/15.pdf

Ministerio de Agricultura, Pesca y Alimentación. (1992). El desarrollo del mundo rural en España (Informe preliminar). Madrid: autor.

Ministerio de Industria, Energía y Turismo. (2012). Plan Nacional e Integral de Turismo 2012-2015. Recuperado de https://www.mincotur.gob.es/turismo/es-ES/PNIT/Documents/Plan\%20Nacional\%20e \%20Integral\%20de\%20Turismo\%20(PNIT)\%202012-2015.pdf

Morrison, F. D. (1987). Multivariate statistical methods. Nueva York: McGraw-Hill.

Navarro, D. (2015). Recursos turísticos y atractivos turísticos: conceptualización, clasificación y valoración. Cuadernos de Turismo, (35), 335-357. https://www.dx.doi.org/10.60I8/turismo.35.22164I

Paniagua, A. (2002). Urban-rural migration, tourism entrepreneurs and rural restructuring in Spain. Tourism Geographies, 4(4), 349-37I. https://www.dx.doi.org/10.1080/I4616680210158128

Panyik, E., Costa, C., y Rátz, T. (20II). Implementing integrated rural tourism: An event-based approach. Tourism Management, 32(6), I352-1363. https://www.dx.doi.org/I0.I0I6/j.tourman.2011.01.009

Parada, A. F., y Rodríguez, J. (2008). Economic valuation of Parque Nacional El Gúacharo, Monagas state, Venezuela. Revista Científica UDO Agrícola, 8(I), 88-97. Recuperado de https://www.dialnet.unirioja.es/s ervlet/articulo?codigo $=3094839$ 
Pérez, C., y Zizumbo, L. (2014). Turismo rural y comunalidad: impactos socioterritoriales en San Juan Atzingo, Mexico. Cuadernos de Desarrollo Rural, iI(73), i7-38. Recuperado de https://www.revistas.javeriana.edu.co /index.php/desarrolloRural/article/view/6ro8/7689

Pérez, S. (2010). El valor estratégico del turismo rural como alternativa sostenible de desarrollo territorial rural. Agronomía Colombiana, 28(3), 507-513. Recuperado de https://www.revistas.unal.edu.co/index.php/agroco l/article/view/I4688/28963

Pitarch, M. D., Buciega, A., y Esparcia, J. (2004). El turismo integrado: repercusiones sociales en áreas rurales. En A. Álvarez-Sousa (coord.), Actas del VIII Congreso Español de Sociología: Turismo, Ocio y Deporte (pp. 247-262). Alicante. Recuperado de https://www.dialnet.unirioja.es/servlet/libro?codigo=6I4I

Plaza, J. I., y Araque, E. (2000). El turismo rural como fuente de desarrollo sostenible: best practices. Revista de Economía y Finanzas de Castilla y León, (4), 77-100.

Pulido, J. I., y Cárdenas, P. J. (20II). El turismo rural en España. Orientaciones estratégicas para una tipología aún en desarrollo. Boletín de la Asociación de Geógrafos Españoles, (56), 155-176. Recuperado de https://www.d ialnet.unirioja.es/servlet/articulo?codigo $=3722434$

Rico, M. (2005). El turismo como nueva fuente de ingresos para el medio rural de Castilla y León. Cuadernos de Turismo, (I6), 175-195. Recuperado de https://www.revistas.um.es/turismo/article/view/18331/1769I

Rocha, F., y Aragón, J. (2012). La crisis económica y sus efectos sobre el empleo en España. Gaceta Sindical: Reflexión y Debate, (19), 67-90.

Schejtman, A., y Berdegué, J. A. (2004). Desarrollo territorial rural. Debates y Temas Rurales (Rimisp), (I). Recuperado de https://www.rimisp.org/wp-content/files_mf/1363093392schejtman_y_berdegue2004_desarr ollo_territorial_rural_5_rimisp_CArdumen.pdf

Secretaría de Estado de Comercio, Turismo y de la Pequeña y Mediana Empresa, y Ministerio de Economía. (2000). Plan Integral de Calidad del Turismo Español (Picte).

Simao, J. N., y Partidario, M. R. (2012). How does tourism planning contribute to sustainable development? Sustainable Development, 20(6), 372-385. https://www.dx.doi.org/10.1002/sd.495

Soret, P. (1999). Turismo rural y de naturaleza. En F. Bayón (dir.), 50 años del turismo español: un análisis histórico y estructural. Madrid: Secretaría de Estado de Comercio, Turismo y Pequeña y Mediana Empresa, Centro de Estudios Ramón Areces.

Swarbrooke, J. (1996). Towards the development of sustainable rural turism in eastern euro. En G. Richards (ed.), Tourism in central and Eastern Europe: Educating for quality (pp. 137-163). Tilburgo: Atlas.

Valdés, L., y del Valle, E. (2003). El turismo rural en España. En Curso Avanzado de Turismo en Áreas Rurales: Estrategias y Promoción. Instituto Agronómico Mediterráneo de Zaragoza.

Varisco, C. A. (2016). Turismo rural: propuesta metodológica para un enfoque sistémico. Revista de Turismo y Patrimonio Cultural, I4(I), I53-I67. Recuperado de https://www.redalyc.org/articulo.oa?id=88I436420II

Yagüe, R. M. (2002). Rural tourism in Spain. Annals of Tourism Research, 29(4), IIOI-IIIO. https://www.doi.org/ 10.1016/S0160-7383(02)00025-7

Zou, T., Huang, S., y Ding, P. (20I2). Toward a community-driven development model of rural tourism: The Chinese experience. International Journal of Tourism Research, I6(3), 26I-27I. https://www.doi.org/10.1002 /jtr.1925

\section{Notas}

* Artículo de investigación

Licencia Creative Commons CC BY-4.० 